\title{
A comparison of the cavitation erosion resistance of TiNi alloys, SUS304 stainless steel and Ni-based self-fluxing alloy
}

\author{
S.K. Wu ${ }^{\mathrm{a}, *}$, H.C. Lin $^{\mathrm{b}}$, C.H. Yeh ${ }^{\mathrm{b}}$ \\ ${ }^{a}$ Institute of Materials Science and Engineering, Department of Mechanical Engineering, National Taiwan University, Taipei, Taiwan 106, China \\ ${ }^{\mathrm{b}}$ Department of Materials Science, Feng Chia University, Taichung, Taiwan 407, China
}

Received 11 October 1999; received in revised form 3 May 2000; accepted 15 June 2000

\begin{abstract}
Cavitation erosion of TiNi shape memory alloys, SUS304 stainless steel (SS) and Ni-based self-fluxing alloys has been investigated in freshwater and $3.5 \mathrm{wt} \% \mathrm{NaCl}$ solution according to the ASTM G32-85 standard method. After 300 min of cavitation erosion, the cumulative weight loss of SUS304 SS is 45 times, whereas that of Ni-based self-fluxing alloy is 15 times the value of TiNi alloys. TiNi alloys and SUS304 SS exhibit a working-hardening behavior in the early cavitation stage, and thereafter maintain constant hardness during further cavitation. However, the Ni-based self-fluxing alloy exhibits no hardening phenomenon during cavitation test. The thermoelastic martensitic transformations of TiNi alloys have important effects on their erosion characteristics. The variants accommodation, pseudoelasticity of SIM and high work-hardening rate can improve the erosion resistance of TiNi alloys. Cavitation erosion of all these alloys in freshwater is similar to that in $3.5 \mathrm{wt}$ \% $\mathrm{NaCl}$ solution under the same testing conditions. (C) 2000 Elsevier Science S.A. All rights reserved.
\end{abstract}

Keywords: Cavitation erosion; TiNi alloys; Infrared joining; Thermoelastic martensitic transformation

\section{Introduction}

TiNi alloys are known as the most important shape memory alloys (SMAs) because of their varied applications based on the shape memory effect (SME) and pseudoelasticity (PE) [1-3]. This is because TiNi alloys have superior properties in ductility, fatigue strength [4], corrosion resistance [5], biocompatibility [6] and recoverable strain [1]. It is also well known that TiNi alloys can exhibit high mechanical damping due to the easy movement of twin boundaries $[7,8]$. In addition to these functional properties, TiNi alloys have recently been found to exhibit excellent wear resistance [9-12], which is ascribed to their pseudoelastic behavior and hardening phenomenon. Suzuki and Kuroyanagi [13] and Shida and Sugimoto [14] also reported that TiNi alloys exhibit a good slurry and water jet erosion resistance in corrosive environment. Hence, TiNi alloys may become a new type of tribo-material in some new applications. In fact, TiNi alloys are expected to have excellent erosion resistance due to their reversible transformation characteristics [15-17], high work-hardening rates [16-20] and high resistance to low-cycle fatigue [21,22]. However, our understand-

\footnotetext{
${ }^{*}$ Corresponding author. Tel.: +886-886-2-2363; fax: +886-886-2-2363-4562.

E-mail address: skw@ccms.ntu.edu.tw (S.K. Wu).
}

ing in this area is incomplete. For example, in the cavitation erosion test of TiNi alloys explosively welded to steel [22], the hardness of TiNi alloys has increased significantly after explosive welding and post-weld heat treatments. This feature indicates that some residual damage has been brought about as a consequence of explosive welding. Another example is the cavitation erosion test of TiNi alloys coated by laser plasma hybrid spraying [23]. The erosion resistance of these TiNi coatings depends strongly on the chemical composition of the coatings and the laser irradiation condition. From the results of Ref. [23], the chemical composition of TiNi coatings is far from the equi-atomic TiNi alloy. Therefore, their SME and PE characteristics should diminish in these TiNi coatings. Obviously, a method which can make TiNi layer clad to steel substrate and still retain its original SME and PE characteristics is necessary. Infrared joining, one of the rapid isothermal processes, can offer a very quick and cost-effective method for joining TiAl intermetallics using $\mathrm{Ti}-15 \mathrm{Cu}-15 \mathrm{Ni}$ foil as brazing filler-metal [24-26]. Our preliminary experiment shows that equi-atomic TiNi alloy can be joined successfully by infrared brazing using Ti-15Cu-15Ni foil without change of its SME and PE properties [27]. In the present study, TiNi shape memory alloys are infrared-brazed to SUS304 stainless steel (SS) by using $\mathrm{Ti}-15 \mathrm{Cu}-15 \mathrm{Ni}$ foil as brazing filler-metal. We aim to investigate the erosion characteristics of TiNi alloys 
Table 1

Transformation temperatures, hardness, and crystal structure at room temperature for TiNi alloys, SUS304 stainless steel and Ni-based self-fluxing alloy

\begin{tabular}{llll}
\hline Alloy & $\mathrm{M}^{\mathrm{a}}\left({ }^{\circ} \mathrm{C}\right)$ & $\mathrm{A}^{\mathrm{b}}\left({ }^{\circ} \mathrm{C}\right)$ & Hardness $(\mathrm{Hv})$ \\
\hline $\mathrm{Ti}_{49} \mathrm{Ni}_{51}$ & -104.4 & -60.1 & 280 \\
$\mathrm{Ti}_{50} \mathrm{Ni}_{50}$ & 34.6 & 78.8 & 214 \\
$\mathrm{Ti}_{50} \mathrm{Ni}_{40} \mathrm{Cu}_{10}$ & $46.6(\mathrm{~B} 2 \rightarrow \mathrm{B} 19)$ & $69.7(\mathrm{~B} 19 \rightarrow \mathrm{B} 2)$ & $\mathrm{B} 2$ parent phase \\
& $6.2\left(\mathrm{~B} 19 \rightarrow \mathrm{B} 19^{\prime}\right)$ & $22.1\left(\mathrm{~B} 19^{\prime} \rightarrow \mathrm{B} 19\right)$ & $\mathrm{B} 19^{\prime}$ martensite \\
$\mathrm{SUS304}$ & - & - & $\mathrm{B} 2{ }^{\prime} \mathrm{B} 19^{\prime} \mathrm{B} 19^{\prime}$ \\
Ni-based-SFA & - & - & 246 \\
\hline
\end{tabular}

${ }^{a}$ DSC peak temperatures of the forward martensitic transformations of TiNi binary and ternary alloys.

${ }^{\mathrm{b}}$ DSC peak temperatures of the reversed martensitic transformations of TiNi binary and ternary alloys.

${ }^{c}$ Composition: $\mathrm{Ni}_{16} \mathrm{Cr}_{4} \mathrm{Si}_{4} \mathrm{~B}_{3} \mathrm{Cu}_{3} \mathrm{Mo}_{2.5} \mathrm{Fe}_{0.5} \mathrm{C}$.

infrared-brazed to SUS304 SS by cavitation tests in both freshwater and $3.5 \mathrm{wt} . \% \mathrm{NaCl}$ solution. The cavitation erosion characteristics of TiNi alloys and some commercial alloys, such as SUS304 SS and Ni-based self-fluxing alloys, are also compared.

In order to investigate the erosion characteristics of $\mathrm{TiNi}$ alloys, it is helpful to first understand some important metallurgical properties of these alloys. Table 1 presents the transformation temperatures, hardness, and crystal structures at room temperature for the $\mathrm{Ti}_{49} \mathrm{Ni}_{51}, \mathrm{Ti}_{50} \mathrm{Ni}_{50}$ and $\mathrm{Ti}_{50} \mathrm{Ni}_{40} \mathrm{Cu}_{10}$ alloys. These properties have no obvious change before, and after, infrared brazing. In addition, the hardness values and crystal structures of SUS304 SS and Ni-based self-fluxing alloy [28] are also presented in Table 1 for comparison. From Table 1, it can be seen that the TiNi alloys and SUS304 SS have similar hardness of about $180-280 \mathrm{Hv}$ and the Ni-based self-fluxing alloy has extra-high hardness of $920 \mathrm{Hv}$. However, it is interesting to find, as presented in Section 3, that TiNi alloys can exhibit a better erosion resistance than the SUS304 SS and Ni-based self-fluxing alloy. Hence, not only the hardness, but also other metallurgical properties, such as phase transformations, work-hardening and resistance to fatigue, will have important influence on the erosion characteristics of TiNi alloys. It is well known that TiNi martensite can be preferentially reoriented to accommodate the deformation strain. Meanwhile, the pseudoelastic behavior originating from the stress-induced martensitic (SIM) transformation of the parent B2 phase can increase the elastic contribution and reduce the plastic deformation during erosion. All these unusual properties of TiNi alloys are considered to be responsible for their high erosion resistance, as discussed in Section 3.

\section{Experimental}

The conventional tungsten arc-melting technique was employed to prepare the $\mathrm{Ti}_{49} \mathrm{Ni}_{51}, \mathrm{Ti}_{50} \mathrm{Ni}_{50}$ and $\mathrm{Ti}_{50} \mathrm{Ni}_{40} \mathrm{Cu}_{10}$ alloys (all in at.\%). Titanium (purity, 99.7 wt.\%), nickel (purity $99.98 \mathrm{wt} . \%$ ) and copper (purity $99.98 \mathrm{wt} . \%$ ), totaling about 200 g, were melted and remelted at least six times in an argon atmosphere. Pure titanium buttons were also melted and used as getters. The mass loss during melting was negligibly small. The as-melted buttons were homogenized at $1050^{\circ} \mathrm{C}$ in a $7 \times 10^{-6}$ torr vacuum furnace for $72 \mathrm{~h}$ and then hot-rolled to about 2-mm thickness plates. Disks of $13 \mathrm{~mm}$ diameter were cut from these plates, annealed at $850^{\circ} \mathrm{C}$ for $2 \mathrm{~h}$, and then both sides of disks were polished to a \#1200 grit finish and well cleaned for the brazing. A Ti-15Cu-15Ni (wt.\%) foil with $50-\mu \mathrm{m}$ thickness (WESGO commercial product with commercial name Ticuni foil) was chosen as the brazing filler metal. Specimens of SUS304 SS with a screw shape and a flat disk head - schematically shown in Fig. 1a - were carefully machined. With similar diameter, a Ticuni foil was sandwiched in between TiNi disk and SUS304 SS specimen's flat head and then brazed by using the ULVAC SINKO-RIKO RHL-P610C type infrared gold
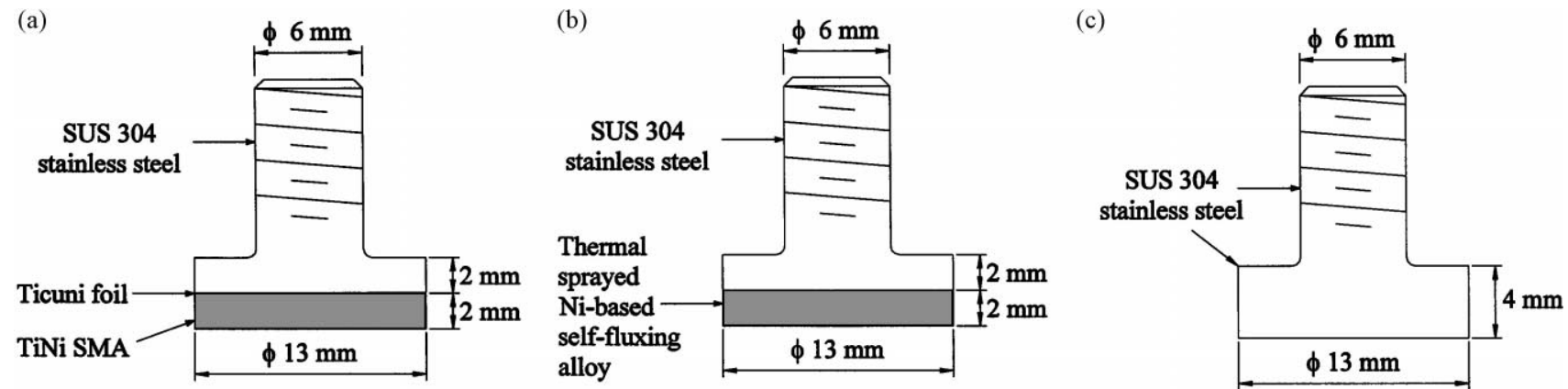

Fig. 1. Configurations and dimensions of cavitation test specimen for (a) TiNi alloys, (b) Ni-based self-fluxing alloy, (c) SUS304 stainless steel. 

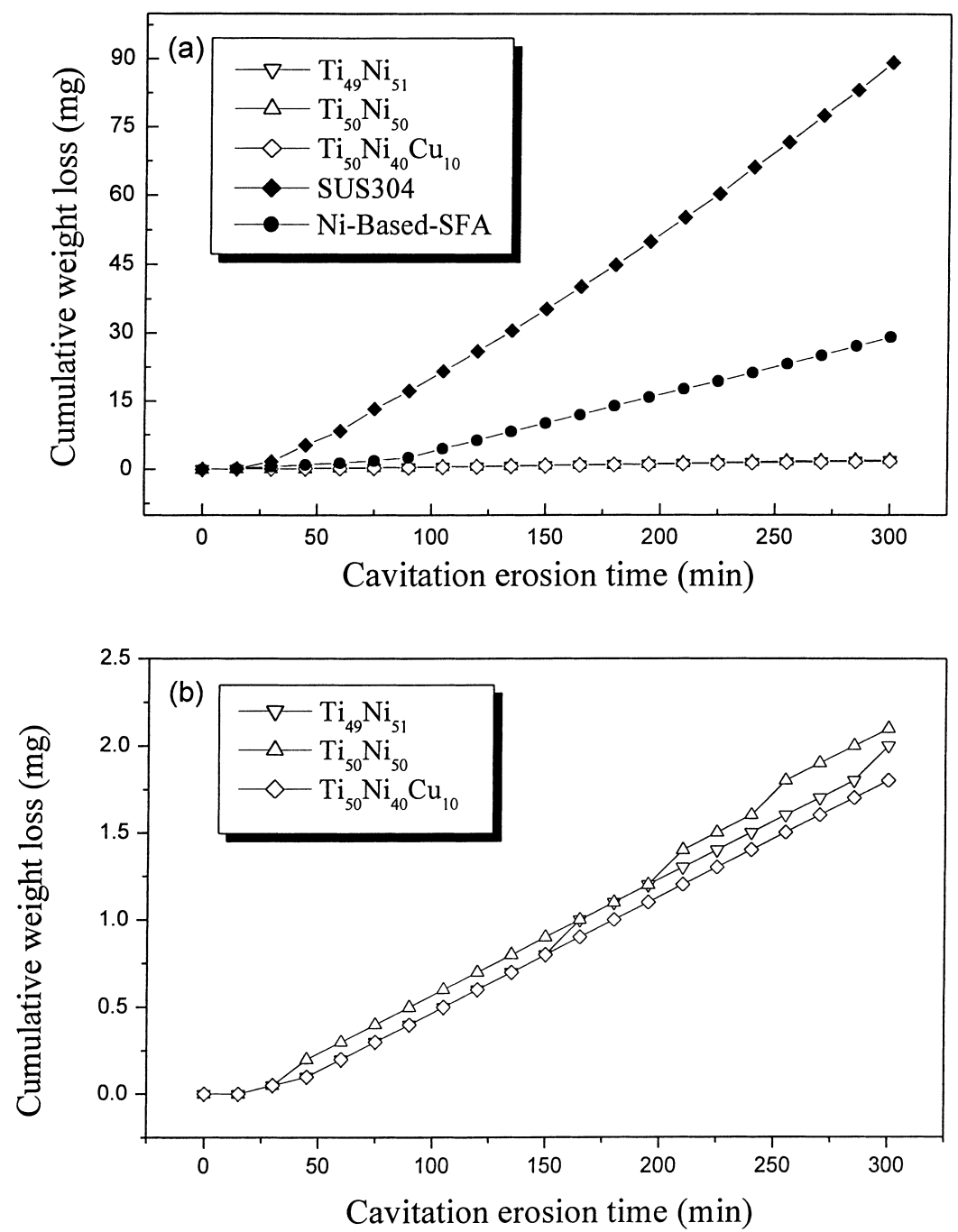

Fig. 2. Cumulative weight loss versus erosion time for (a) all the tested alloys, and (b) TiNi alloys only.

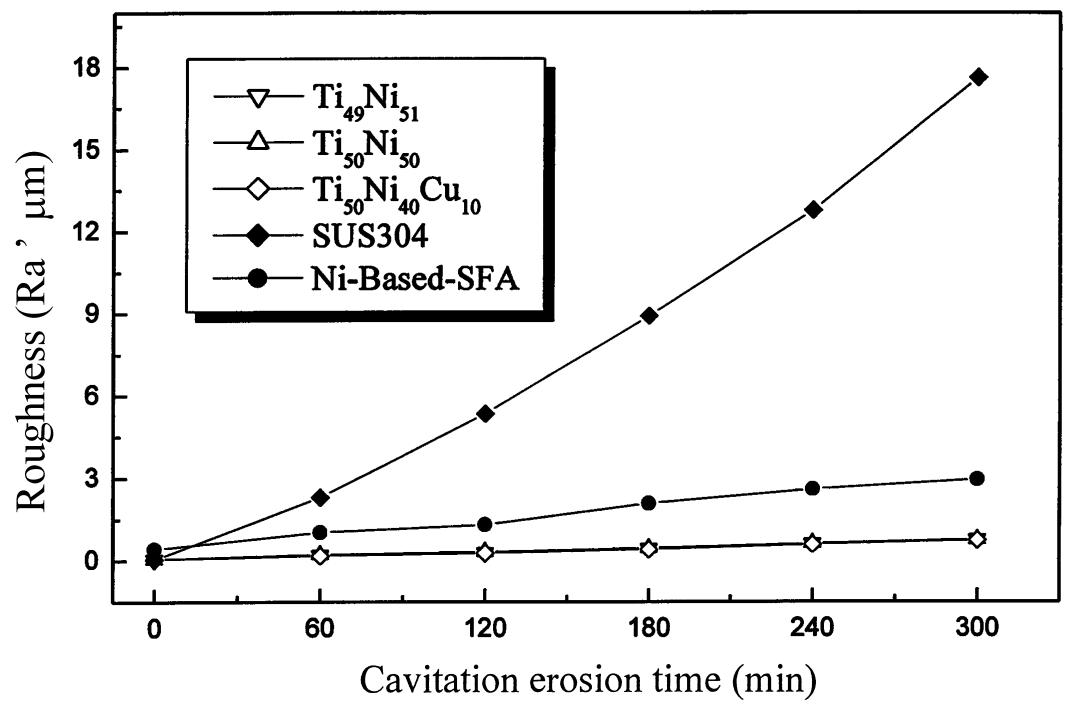

Fig. 3. Surface roughness versus erosion time for the tested samples. 
image furnace. The brazing process was $1050^{\circ} \mathrm{C} \times 30 \mathrm{~s}$. in flowing Ar gas. The detailed procedures of the brazing process have been described elsewhere [25,27]. The test specimens of Ni-based self-fluxing alloy were prepared using the thermal-spraying technique. SUS304 SS was selected as substrate on which thermal sprays of Ni-based self-fluxing alloy were applied with a Metco 5P gun. After a coating of 2-mm thickness had been applied, test pieces shown in Fig. 1b were carefully machined and then fusion-sintered in a vacuum fur-
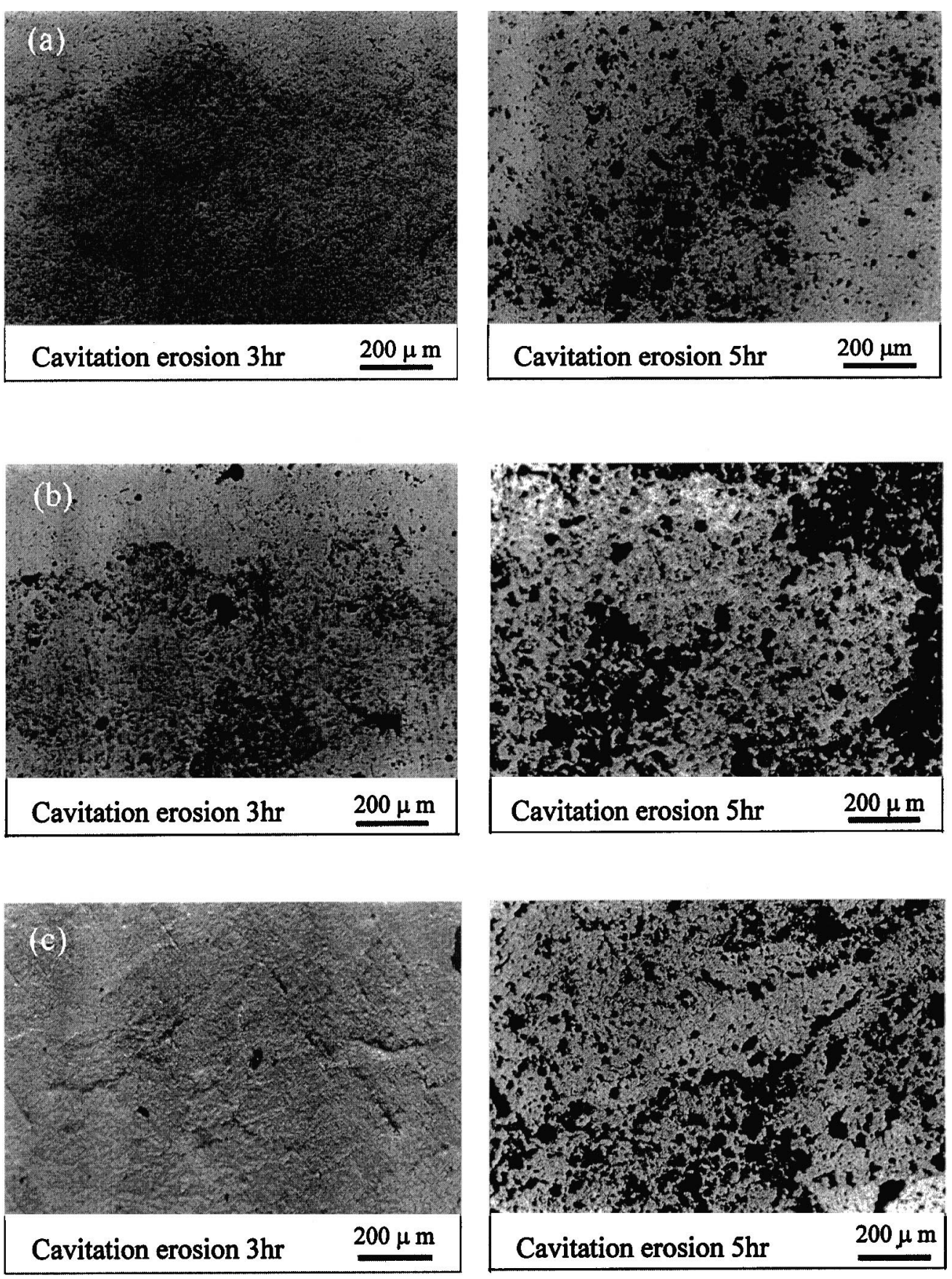

Fig. 4. Optical micrographs of eroded surfaces for (a) $\mathrm{Ti}_{49} \mathrm{Ni}_{51}$, (b) $\mathrm{Ti}_{50} \mathrm{Ni}_{50}$, (c) $\mathrm{Ti}_{50} \mathrm{Ni}_{40} \mathrm{Cu}_{10}$, (d) SUS304 stainless steel, and (e) Ni-based self-fluxing alloy, subjected to various periods of cavitation testing.

nace. The detailed procedures of the thermal spraying and fusion sintering have been described in Ref. [28]. The individual SUS304 SS test specimens were directly machined to the dimension of Fig. 1c. The cavitation test was carried out according to the ASTM G32-85 standard test method [29] in both freshwater and $3.5 \mathrm{wt} . \% \mathrm{NaCl}$ solution. Cavitation was induced by longitudinal oscillations at $20 \mathrm{kHz}$ with an amplitude of $50 \mu \mathrm{m}$. The testing temperature of the medium was $25^{\circ} \mathrm{C}$. 

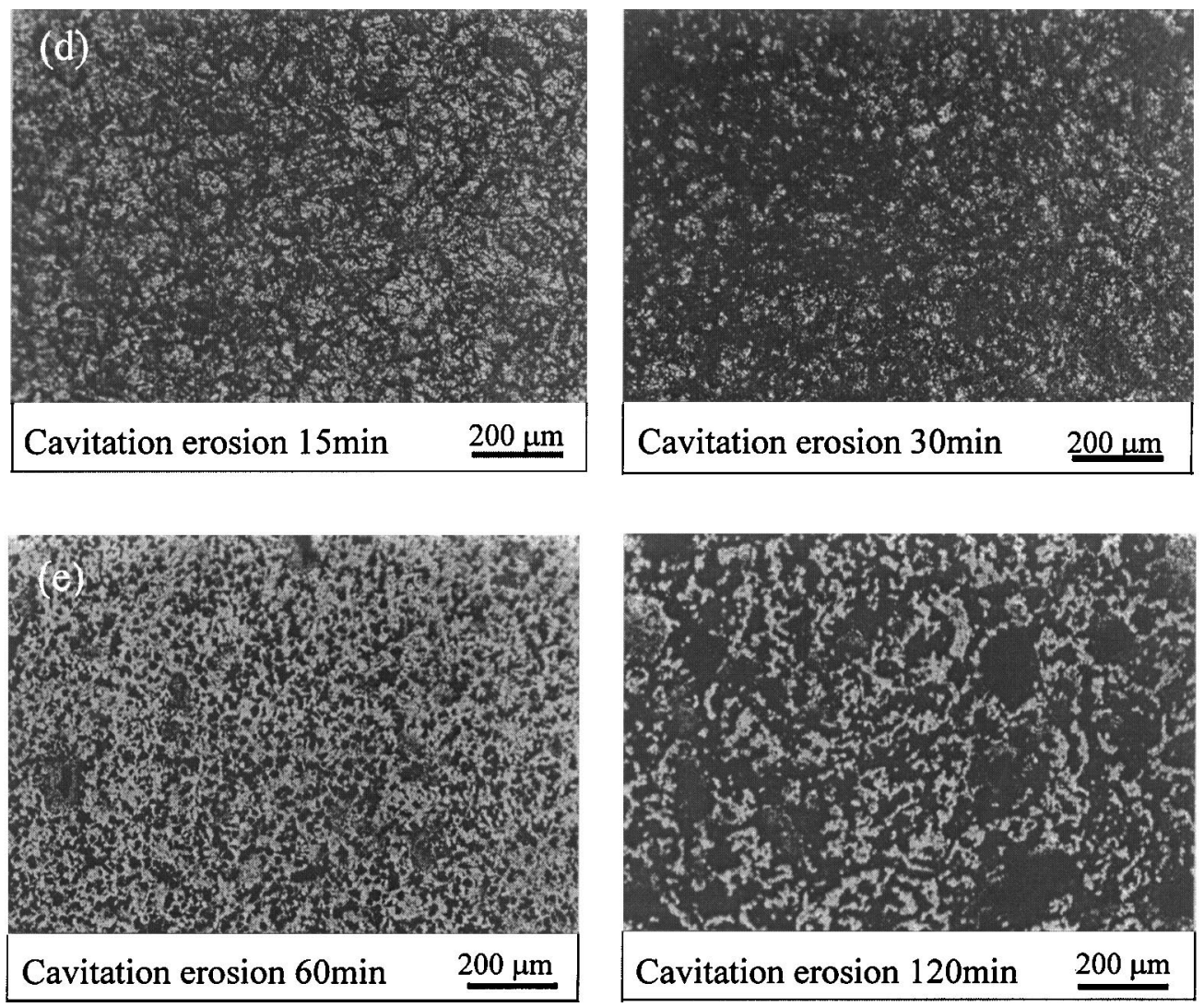

Fig. 4. (Continued).

The microstructures of eroded surfaces were studied by $\mathrm{X}$-ray diffraction (XRD), scanning electron microscopy (SEM) and optical microscope (OM). XRD tests were carried out on a MAC-MXP-3 X-ray diffractometer using $\mathrm{CuK} \alpha$ radiation. The power was $40 \mathrm{kV} \times 30 \mathrm{~mA}$ and the $2 \theta$ scanning rate was $1^{\circ} \mathrm{min}^{-1}$. The surface morphologies were observed by a Topcon ABT-55 SEM and an Olympus OM system. The surface hardness was measured by a Vickers microhardness tester with a load of $100 \mathrm{~g}$ for $15 \mathrm{~s}$. For each specimen, the average hardness value was calculated from at least five test readings. The weight loss after cavitation erosion was measured by using a precise electronic balance, with a weighing accuracy of $\pm 0.01 \mathrm{mg}$. The average roughness, $R_{\mathrm{a}}$, of eroded surface was measured and automatically calculated by using a Hommel Tester T1000, made by THYSSEN Co. in Germany.

\section{Results and discussion}

\subsection{Weight loss and surface roughness after cavitation erosion}

Fig. 2 shows the cumulative weight loss of different samples plotted as a function of cavitation erosion time. In general, all tested specimens exhibit no weight loss at initial erosion time, and this regime is commonly referred to as the incubation period [30]. After the incubation period, the cumulative weight loss generally increases with erosion time. As can be seen in Fig. 2, both SUS304 SS and Ni-based self-fluxing alloy have a rapid erosion rate, especially for the SUS304 SS. However, TiNi alloys exhibit no obvious weight loss even when the erosion time is up to $300 \mathrm{~min}$. This indicates that TiNi alloys are more resistant to cavitation erosion than the SUS304 SS and Ni-based self-fluxing alloys, which are commonly used as erosion-resistant materials in hydraulic machinery. After $300 \mathrm{~min}$ of cavitation erosion, the cumulative weight loss of SUS304 SS is 45 times, while that of Ni-based self-fluxing alloy is 15 times the value of the TiNi alloys.

Fig. 3 shows the surface roughness versus erosion time for the tested samples. A comparison between Figs. 2 and 3 shows that the variation tendency of cumulative weight loss and that of surface roughness versus erosion time is similar. Hence, the SUS304 SS has the highest roughness and cumulative weight loss, while TiNi alloys have the lowest cumulative weight loss and the smoothest eroded surface. It should be mentioned that all the tested specimens were mechanically polished to a \#1200 grit $\mathrm{SiC}$ paper. However, the Ni-based self-fluxing alloy exhibits a little higher surface roughness than the TiNi alloys. This feature is ascribed to the retained porosities formed during the thermal spraying of 
Ni-based self-fluxing alloy on SUS304 SS substrate. These retained porosities are considered to have some effect on the erosion behavior of Ni-based self-fluxing alloys. This phenomenon will be further elaborated in the following.

\subsection{Morphologies of eroded surfaces}

Fig. 4a-e are optical micrographs of the eroded surfaces for $\mathrm{Ti}_{49} \mathrm{Ni}_{51}, \mathrm{Ti}_{50} \mathrm{Ni}_{50}, \mathrm{Ti}_{50} \mathrm{Ni}_{40} \mathrm{Cu}_{10}, \mathrm{SUS} 304 \mathrm{SS}$ and Ni-based self-fluxing alloy, respectively, subjected to various periods of cavitation testing. In Fig. $4 a-c$, only a slight damage trace appears for TiNi alloys subjected to $3 \mathrm{~h}$ of cavitation erosion. After that, the damage trace gradually increases, because the damage pits pre-formed at early cavitation grow during further cavitation erosion. However, for both SUS304 SS and Ni-based self-fluxing alloy, an obvious damage trace has been observed after a short period of cavitation erosion and it increases rather rapidly during further cavitation erosion, as shown in Fig. 4d-e. As mentioned above, porosities are retained within the Ni-based
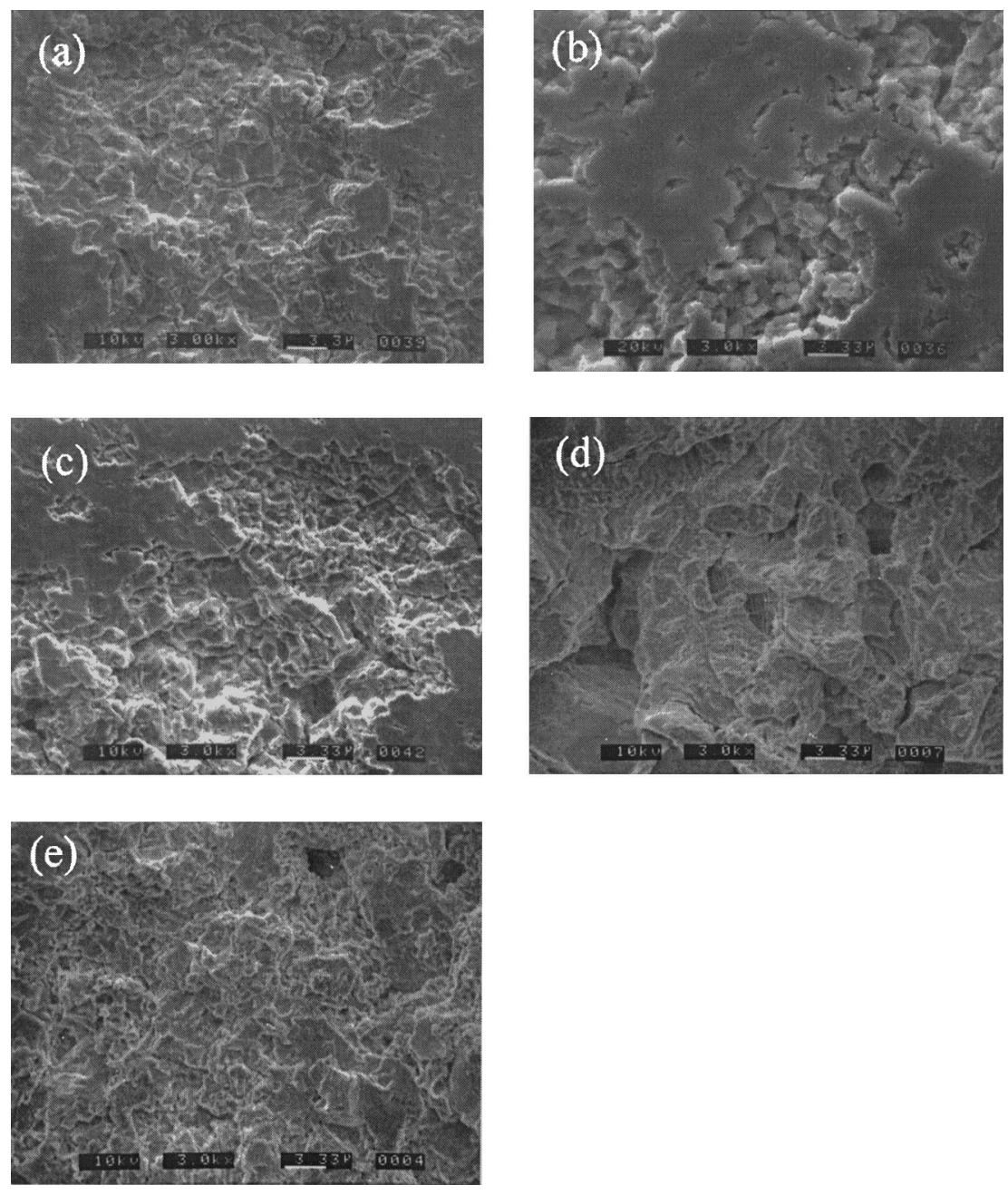

Fig. 5. Scanning electron micrographs of eroded surfaces for (a) $\mathrm{Ti}_{49} \mathrm{Ni}_{51}$, (b) $\mathrm{Ti}_{50} \mathrm{Ni}_{50}$, (c) $\mathrm{Ti}_{50} \mathrm{Ni}_{40} \mathrm{Cu}_{10}$, (d) SUS304 stainless steel, and (e) Ni-based self-fluxing alloy, subjected to cavitation testing for $5 \mathrm{~h}$. 
surface as shown in Fig. 5d. In Fig. 5e, the Ni-based self-fluxing alloy exhibits an eroded surface similar to that of SUS304 SS, except that its damage pits are smaller. The eroded surfaces of these alloys shown in Figs. 4 and 5 are consistent with the variation in weight loss after cavitation erosion test. The TiNi alloys are more resistant to cavitation erosion than the SUS304 SS and Ni-based self-fluxing alloy. It is worth mentioning that the surface microstructures of TiNi alloys after cavitation erosion show no obvious change, though a small variation in XRD peak intensity occurs, as shown in Fig. 6. This indicates that the martensite-variants accommodation and the pseudoelasticity of SIM of TiNi alloys can effectively accommodate the impact stress and deformation strain during cavitation erosion.

\subsection{Hardness of eroded surfaces}

Fig. 7 shows the plot of surface hardness versus erosion time for the tested samples. In Fig. 7, the Ni-based self-fluxing alloy exhibits no hardening phenomenon. This feature is ascribed to its high hardness and brittle behavior. Due to its high hardness and wear resistance, the Ni-based self-fluxing alloy is expected to exhibit a reasonably good erosion resistance. However, TiNi alloys and SUS304 SS, being ductile materials, will induce lots of crystal defects during cavitation erosion and exhibit an obvious work hardening behavior in the first 15 min of cavitation erosion, and then maintain constant hardness during further cavitation, as shown in Fig. 7. After a micrometer-order thickness of the eroded surface is polished, the surface hardness resumes the same value as the un-eroded specimen. This indicates that work hardening only occurs at a thin surface layer. The cavitation erosion is a steady-state damage process. The material surface, being subjected to continuous impact, is continuously work-hardened and then fractured. Hence, this thin work-hardened layer could have important contributions to the erosion resistance.

On a careful examination, TiNi alloys exhibit a higher work-hardening rate than SUS304 SS (Fig. 7). This phenomenon can also be observed from the stress-strain curves shown in Fig. 8, where the work-hardening rate in the region III of TiNi alloys is higher than that in the region $\mathrm{V}$ of SUS304 SS. As mentioned in Section 1, materials with higher work-hardening rate would exhibit better erosion resistance [16-20]. Hence, the work-hardening characteristic of TiNi alloys will provide an important contribution to their high erosion resistance. This phenomenon is further discussed in the following.

As can be seen in Fig. 8, TiNi alloys exhibit a lower yielding stress than SUS304 SS. But they can exhibit a large reversible transformation strain, which is associated with the elastic strain, variants accommodation and pseudoelasticity. The first erosive impact will be partially accommodated by the martensite variants and stress-induced martensite, and then introduces a limited amount of work-hardening. During the further erosive impact, a higher impact stress
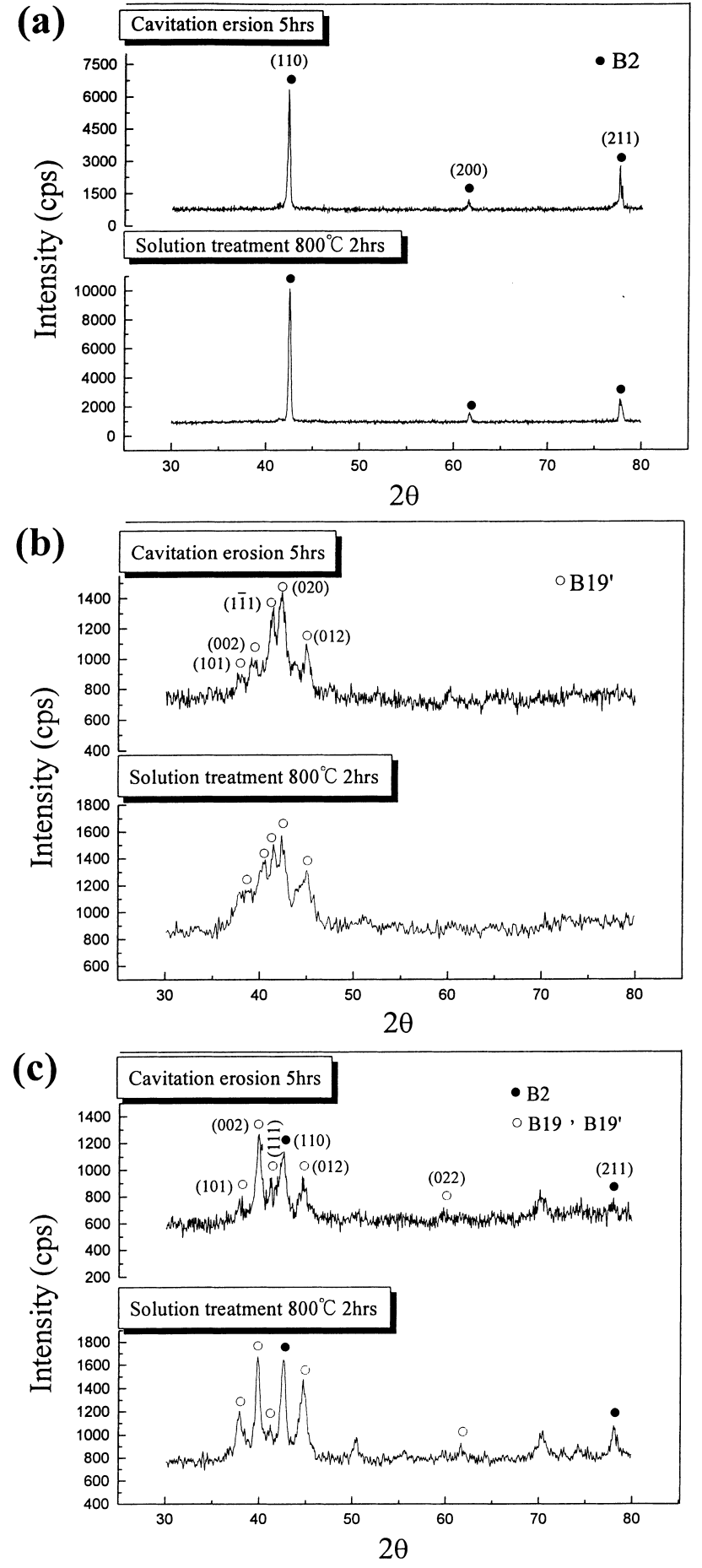

Fig. 6. The X-ray diffraction of eroded surfaces for (a) $\mathrm{Ti}_{49} \mathrm{Ni}_{51}$, (b) $\mathrm{Ti}_{50} \mathrm{Ni}_{50}$, and (c) $\mathrm{Ti}_{50} \mathrm{Ni}_{40} \mathrm{Cu}_{10}$.

is necessary to cause further work hardening, and hence the quantity of effective impact of higher stress level becomes smaller. This feature can elucidate why materials with higher work-hardening rate would exhibit better erosion resistance. As can be seen in Fig. 8, TiNi alloys can exhibit a reversible transformation strain up to $8 \%$, and have a high work-hardening rate. These reversible transformation strains dissipate the impact energy and obviate the 


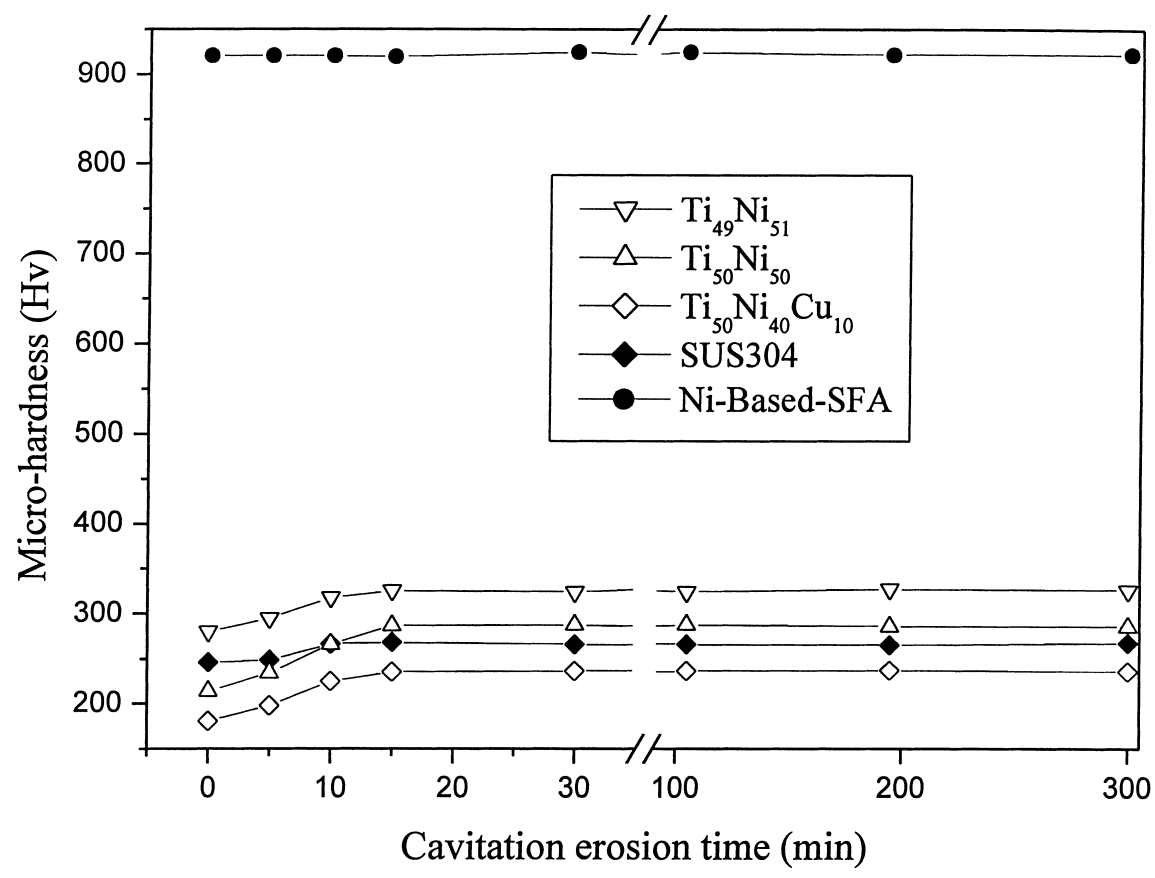

Fig. 7. Surface hardness versus erosion time for the tested samples.

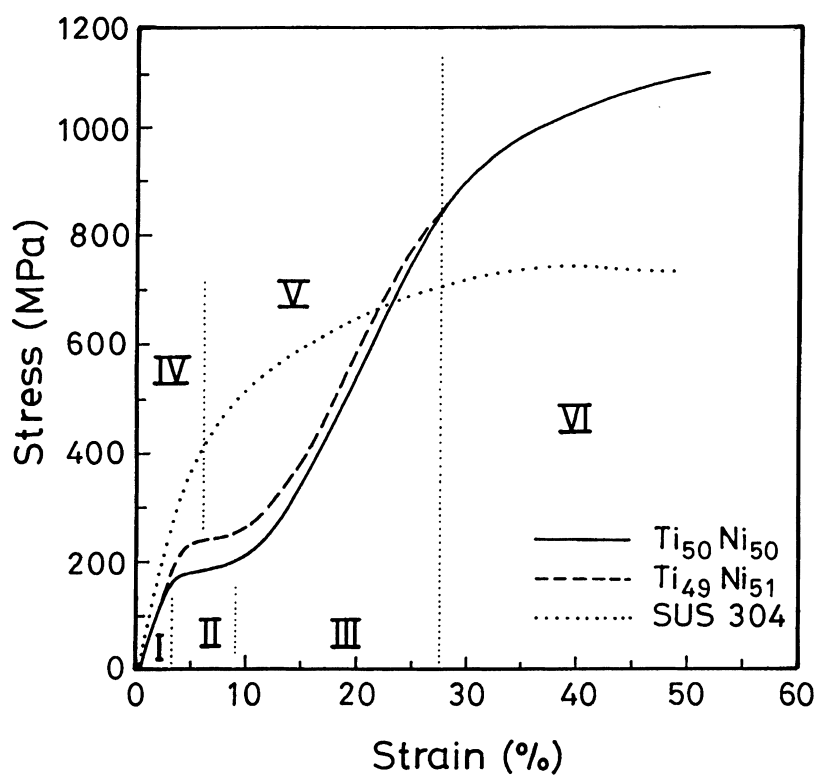

I : elastic region of TiNi alloys

II : pseudoelastic or variants accomodation region of TiNi alloys

\section{III : work-hardening region of TiNi alloys \\ IV : elastic region of SUS304 SS \\ $\mathrm{V}$ : work-hardening region of SUS304 SS \\ VI : full work-hardening region for all alloys}

Fig. 8. Stress-strain curves of $\mathrm{Ti}_{50} \mathrm{Ni}_{50}, \mathrm{Ti}_{49} \mathrm{Ni}_{51}$ and $\mathrm{SUS} 304$ stainless steel. possibility of brittle fracture and spalling. Meanwhile, the characteristic of high work-hardening rate can reduce the chance of impact loss for a given spectrum of erosive impact and the lifetime of a surface can be prolonged. Hence, they can exhibit an excellent erosion resistance.

It is worth mentioning that the erosion results, involving weight loss, surface roughness, hardness and morphologies of eroded surface, are all nearly the same for cavitation tests in both freshwater and $3.5 \mathrm{wt} \% \mathrm{NaCl}$ solution for these five alloys. This phenomenon can be ascribed to all these alloys having an excellent corrosion resistance; hence, no extra corrosive effect would occur during cavitation erosion in $3.5 \mathrm{wt} . \% \mathrm{NaCl}$ solution.

\subsection{Effects of thermoelastic martensitic transformations on cavitation erosion of TiNi alloys}

It is important to establish the erosion characteristics of TiNi alloys and understand why they have high cavitation erosion resistance despite their low hardness. The unusual erosion characteristics are believed to be related to their thermoelastic behavior, fatigue performance and work-hardening characteristics. During cavitation erosion of TiNi martensite, partial impact energy is absorbed by variants accommodation. In the parent $\mathrm{B} 2$ phase of $\mathrm{TiNi}$ alloys, the applied strain during cavitation erosion will be mostly transformed to pseudoelastic strain due to the formation of stress-induced martensite (SIM). This SIM will resume their parent phase after the relief of impact load. These transformation strains induced by erosive impacts are mostly reversible and, thus, the material surface would have an indefinite lifetime. Hence, the martensite-variants 
accommodation and SIM formation of TiNi alloys will provide an important contribution to their erosion characteristics. Meanwhile, the fatigue crack tips can also be blunted and stabilized due to stress relief by the variants accommodation and/or pseudoelasticity. For most metals and alloys, plastic deformation and/or crack propagation release the strain energy of crack tips. However, for TiNi alloys, the variants accommodation and/or pseudoelasticity will absorb partial impact energy to be stored in the martensite variants and/or SIM. These features will stabilize the fatigue crack tips and hinder crack propagation, hence improving the erosion characteristics of TiNi alloys. If the transformation strain associated with the variants accommodation and/or pseudoelasticity is exceeded, then high work-hardening occurs and thus resistance to cavitation erosion.

\section{Conclusions}

1. TiNi alloys are more resistant to cavitation erosion than the SUS304 SS and Ni-based self-fluxing alloys which are commonly used as erosion-resistant materials in hydraulic machinery. After 300 min of cavitation erosion test, the cumulative weight loss of SUS304 SS is 45 times, while that of Ni-based self-fluxing alloy is 15 times the value of TiNi alloys.

2. TiNi alloys exhibit a lower yielding stress, but a higher work-hardening rate, than SUS304 SS. These features can absorb more impact energy and reduce the material loss for a given spectrum of erosive impact, and hence improve the erosion resistance of TiNi alloys. Owing to its high hardness and brittle behavior, the Ni-based self-fluxing alloy exhibits no hardening phenomenon during cavitation testing.

3. The thermoelastic martensitic transformation of TiNi alloys has important effects on their erosion characteristics. The variants accommodation and pseudoelasticity will absorb partial impact energy to be stored in the martensite variants and SIM. If the reversible transformation strain is exceeded, then a high work-hardening rate occurs. These features will also stabilize the fatigue crack tips and hinder crack propagation, hence improving the erosion resistance of TiNi alloys.

4. The characteristic of cavitation erosion of TiNi alloys, SUS304 SS and Ni-based self-fluxing alloy in freshwater is similar to that in $3.5 \mathrm{wt} \% \mathrm{NaCl}$ solution under the same testing condition. This is ascribed to the fact that all these alloys have an excellent corrosion resistance in 3.5 wt. $\% \mathrm{NaCl}$.

\section{Acknowledgements}

The authors are pleased to acknowledge the financial support for this research by the National Science Council (NSC),
Republic of China, under Grants NSC 87-TPC-E002-022 and NSC 88-2216-E002-012. The authors also express their sincere appreciation to Profs. J.L. He and K.M. Lin, Department of Materials Science, Feng Chia University, Taichung, Taiwan, for their kind assistance with the use of experimental equipment, and Dr. Y.H. Shieh, Electrical Power Research Institute, Taiwan Power Company, Taipei, Taiwan, for his kind provision of the Ni-based self-fluxing alloy.

\section{References}

[1] C.M. Jackson, H.J. Wagner, R.J. Wasilewski, NASA Report SP-5110 (1972).

[2] G.D. Sandrock, A.J. Perkins, R.F. Hehemann, Metall. Trans. A 2 (1971) 2769.

[3] S. Miyazaki, T. Imai, Y. Igo, K. Otsuka, Metall. Trans. A 17 (1986) 115-120.

[4] K.N. Melton, O. Mercier, Acta Metall. 27 (1979) 137-144.

[5] Y. Oshida, S. Miyazaki, Corr. Eng. 40 (1991) 1009-1025.

[6] L.S. Castleman, S.M. Motzkin, in: D.F. Williams (Ed.), Biocompatibility of Clinical Implant Materials, CRC Press, Boca Raton, FL, 1981, pp. 129-154.

[7] H.C. Lin, S.K. Wu, M.T. Yeh, Metall. Trans. A 24 (1993) 21892194.

[8] H.C. Lin, S.K. Wu, Y.C. Chang, Metall. Mater. Trans. A 26 (1995) 851-858.

[9] J.L. Jin, H.L. Wang, Acta Metall. Sinica 24 (1988) A66-69.

[10] D.Y. Li, Scripta Metall. 34 (1996) 195-200.

[11] P. Clayton, Wear 162-164 (1993) 202-210.

[12] H.C. Lin, H.M. Liao, J.L. He, K.C. Chen, K.M. Lin, Metall. Mater. Trans. A 28 (1997) 1871-1877.

[13] Y. Suzuki, T. Kuroyanagi, FAEDIC-NT, Titanium Zirconium 27 (1979) 67-73.

[14] Y. Shida, Y. Sugimoto, Wear 146 (1991) 219-228.

[15] E. Nakao, S. Hattori, Proc. Jpn. Soc. Mech. Eng. (in Japanese) 64 (1998) 2555-2560.

[16] A. Ball, Wear 91 (1983) 201-207.

[17] C.J. Heathcock, B.E. Protheroe, A. Ball, Proceedings of the 5th International Conference on the Strength of Metals and alloys, Pergamon Press, Oxford, 1979, pp. 219-224.

[18] A.W.J. de Gee, Wear 81 (1982) 373.

[19] W.J. Salesky, G. Thomas, Wear 75 (1982) 21.

[20] C. Allen, A. Ball, B.E. Protheroe, Wear 74 (1981-1982) 287.

[21] R.H. Richman, A.S. Rao, D. Kung, Wear 157 (1992) 401-407.

[22] R.H. Richman, W.P. McNaughton, J. Mater. Eng. Perfor. 6 (1997) 633-641.

[23] H. Hiraga, T. Inoue, H. Shimura, A. Matunawa, Wear 231 (1999) 272-278.

[24] C.A. Blue, R.A. Blue, R.Y. Lin, Scripta Metall. Mater. 32 (1988) $127-132$.

[25] S.J. Lee, S.K. Wu, R.Y. Lin, Acta Mater. 46 (1998) 1283-1295.

[26] S.J. Lee, S.K. Wu, R.Y. Lin, Acta Mater. 46 (1998) 1297-1305.

[27] T.Y. Yang, S.K. Wu, unpublished.

[28] Y.H. Shieh, J.T. Wang, H.C. Shih, S.T. Wang, Surface Coatings Technol. 58 (1993) 73-77.

[29] ASTM G32-85, Standard Method of Vibratory Cavitation Erosion Test, ASTM, 1985.

[30] G.C. Gould, in: A. Thiruvengadam, F.J. Heymann (Eds.), Characterization and Determination of Erosion Resistance, ASTM STP414, ASTM, Philadelphia, PA, 1970, pp. 182-211. 